\title{
Asymmetrically coupled directed percolation systems
}

\author{
Jae Dong Noh \\ Department of Physics, Chungnam National University, Daejeon 305-764, Korea \\ Hyunggyu Park \\ School of Physics, Korea Institute for Advanced Study, Seoul 130-722, Korea
}

(Dated: December 4, 2018)

\begin{abstract}
We introduce a dynamical model of coupled directed percolation systems with two particle species. The two species $A$ and $B$ are coupled asymmetrically in that $A$ particles branch $B$ particles whereas $B$ particles prey on $A$ particles. This model may describe epidemic spreading controlled by reactive immunization agents. We study nonequilibrium phase transitions with focused attention to the multicritical point where both species undergo the absorbing phase transition simultaneously. In one dimension, we find that the inhibitory coupling from $B$ to $A$ is irrelevant and the model belongs to the unidirectionally coupled directed percolation universality class. On the contrary, a mean field analysis predicts that the inhibitory coupling is relevant and a new universality appears with a variable dynamic exponent. Extensive numerical simulations on small-world networks confirm our predictions.
\end{abstract}

PACS numbers: 64.60.Ht,05.70.Ln,05.40.-a,89.75.Da

It is a challenging problem to establish the concept of the universality class in nonequilibrium phase transitions. In contrast to equilibrium systems, nonequilibrium systems lack a general framework to begin with and display too diverse critical phenomena to be classified into a few universality classes. Recently the absorbing phase transition has been attracting much theoretical interest since the universality class concept can be applied. It is a phase transition from an inactive (dead) phase into an active (live) phase, which may occur in systems with microscopic trapped (absorbing) states which the system, once trapped, cannot excape out of.

Absorbing critical phenomena are categorized into a few universality classes depending on conservation in dynamics and symmetry between absorbing states (see for review Ref. [1]). The directed percolation (DP) class is the most well-established one, which encompasses a vast number of systems [2, 3] . The contact process [4] is an archetype of the DP class. In this model, each lattice site is either empty ( $\emptyset)$ or occupied by a particle $(X)$. Each particle may annihilate spontaneously $(X \rightarrow \emptyset)$ or branch an offspring to a neighboring site $(X \rightarrow X X)$. The contact process was originally suggested as a model for epidemic spreading without immunization [4], where the particle represents an infected individual, who may recover spontaneously or infect its healthy neighbors. The vacuum state with all sites empty is the unique absorbing state, which is one of the key ingredients in the DP class.

Recently, coupled DP systems have been studied extensively [5, 6, 7, 8]. In particular, the unidirectionally coupled DP (UCDP) process introduced by Täuber et al. [7] was found to display a series of new multicritical phenomena. In the UCDP process, there are an infinite number of particle species $X_{k}$ with $k=0,1,2, \cdots$ with dynamic rules characterized by $X_{k} \rightarrow \emptyset, X_{k} \rightarrow X_{k} X_{k}$, and $X_{k} \rightarrow X_{k} X_{k+1}$. The coupling is unidirectional (upward), linear, and excitatory. The species $X_{0}$ is not affected by the others, hence its absorbing critical phenomena belong to the DP class. The other species $X_{k>0}$, however, are dressed by the critical fluctuations of particles of species $X_{k^{\prime}<k}$ at the multicritical point where all species undergo the absorbing phase transition simultaneously. It turned out that their critical behaviors are described by the critical exponents distinct from those of the DP class [7, 8]. This novel critical behavior is destroyed and the DP scaling is recovered as soon as we turn on the excitatory coupling in the other (downward) direction [6, 8].

It is noteworthy that the UCDP process was proposed for describing an interesting roughening transition in an onedimensional growth process [9], where the monomer-type restricted-solid-on-solid (RSOS) model is considered with the constraint that evaporation is allowed only at the edges (not terraces). It can be easily shown that the growth model is mapped to the UCDP process, except that the RSOS condition generates an extra inhibitory coupling in the downward direction, i.e. the offspring production of $X_{k}$ is suppressed by the presence of $X_{k^{\prime}}$ for $k^{\prime}>k$ (see Sec.VA in [8]). The coupling should be at least bilinear, so the simplest process one may consider is $X_{k} X_{k+1} \rightarrow X_{k+1}$. Numerical studies [8, 9] revealed that the one-dimensional growth model displays the same critical behavior as the UCDP, which may imply the irrelevance of the inhibitory coupling in coupled DP processes.

In this Letter, we study a coupled DP system with two particle species, which has a linear excitatory coupling in one direction and a bilinear inhibitory coupling in the other direction. We show that the inhibitory coupling is irrelevant only in low dimensions (UCDP class) but becomes relevant in high dimensions, at least where the mean field theory becomes valid. Our model may also serve as a prototype model for epidemic spreading process in the presence of reactive immunization agents, which will be explained shortly.

We consider a coupled DP system with two particle species $A$ and $B$ on lattices or networks in general. Specifically we choose the contact process dynamics for both species in the 


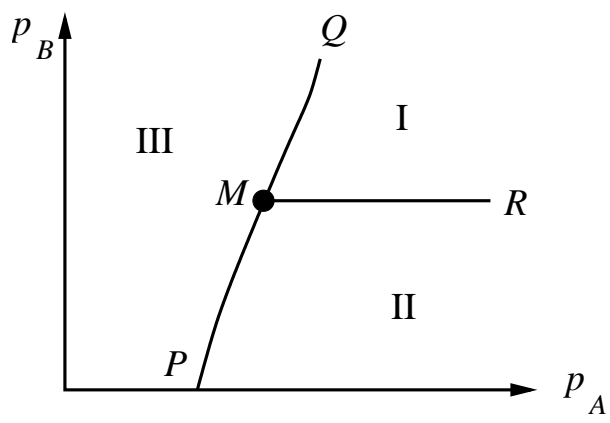

FIG. 1: A schematic phase diagram of the ACDP process in the $\left(p_{A}, p_{B}\right)$ plane with fixed $\lambda$ and $\mu$.

noninteracting limit; each particle annihilates spontaneously or branches one offspring of the same kind to a neighboring site. The two species are coupled asymmetrically through branching and predation processes, that is, an $A$ particle branches a $B$ particle to a neighboring site (excitatory coupling), while a $B$ particle preys on an $A$ particle at the same site (inhibitory coupling).

The dynamic rules are summarized as follows: (i) $A(B) \rightarrow$ $\emptyset$ with probability $p_{A}\left(p_{B}\right)$, (ii) $A \rightarrow A A$ with probability $(1-$ $\left.p_{A}\right)(1-\lambda)$, (iii) $B \rightarrow B B$ with probability $1-p_{B}$, (iv) $A \rightarrow A B$ with probability $\left(1-p_{A}\right) \lambda$, and (v) $A B \rightarrow B$ with probability $\mu$. The order parameters for the phase transition are the particle densities, $\rho_{A}$ and $\rho_{B}$. The model will be referred to as the asymmetrically coupled DP (ACDP) process. When $\mu=0$, the coupling becomes unidirectional and the model reduces to the two-species case of the UCDP process.

In the context of epidemiology, $A$ particles correspond to antigens (or viruses) and $B$ particles correspond to antibodies (or vaccines). Both antigens and antibodies can replicate themselves and may die out by themselves (processes (i), (ii), and (iii)). In addition, an antigen promotes an antibody (iv) and can be destroyed by the antibody (v).

A schematic phase diagram of the ACDP process is shown in Fig. 11 In the phase I where both annihilation probabilities are large, both species are inactive with $\rho_{A}=\rho_{B}=0$ in the steady state. When $p_{B}$ is smaller, the $B$ species becomes active with the $A$ species remaining inactive. Hence, in the phase II, $\rho_{A}=0$ and $\rho_{B} \neq 0$ in the steady state. When $p_{A}$ is small, the $A$ species is active, so is the $B$ species regardless of the value of $p_{B}$ due to the process $A \rightarrow A B$. So, both species are active with $\rho_{A} \neq 0$ and $\rho_{B} \neq 0$ in the phase III. Since $\rho_{A}=0$ throughout the phases I and II, the phase boundary line $\overline{M R}$ should be flat with $p_{B}=p_{B}^{c}$ which is a constant independent of $p_{A}, \lambda$, and $\mu$, and is identical to the critical probability of the uncoupled system.

The nature of the phase transitions is easily inferred. Across the line $\overline{M R}$, the $B$ species undergoes the absorbing transition with $\rho_{A}=0$. Across the line $\overline{M P}$, the $A$ species undergoes the transition while $\rho_{B}$ remains finite. Across the line $\overline{M Q}$, both species undergoes the transition with $\rho_{B} \propto \rho_{A}$ since the $B$ particles are mostly slaved by the $A$ particles through the $A \rightarrow$
$A B$ process for large $p_{B}$ region. Therefore, one may easily expect that the three critical lines should belong to the DP class. That was confirmed by numerical simulations, which we do not present here. Interesting critical phenomena can be observed along a line through the multicritical point $M$ from the phase III to the phase I. In this Letter, we determine the location of $M$ with high precision and mainly focus on density decay dynamics of both species at $M$, which may display a novel critical behavior distinct from the DP and also from the UCDP.

First, we investigate the model using the mean field theory which assumes that local densities of $A$ and $B$ particles are uniform and uncorrelated spatially with the mean values $\rho_{A}$ and $\rho_{B}$, respectively. The mean field rate equations can be written as

$$
\begin{aligned}
& \dot{\rho}_{A}=a_{A} \rho_{A}-\rho_{A}^{2}-\widetilde{\mu} \rho_{A} \rho_{B}, \\
& \dot{\rho}_{B}=a_{B} \rho_{B}-\rho_{B}^{2}+\widetilde{\lambda} \rho_{A},
\end{aligned}
$$

where the densities are rescaled to fix the coefficient of the $\rho^{2}$ terms to be unity and the parameters are functions of the model parameters. Higher order terms are neglected.

The stationary state densities are obtained by solving Eq. (1) with $\dot{\rho}_{A}=\dot{\rho}_{B}=0$ and hence the phase diagram is determined. It has the same structure as shown in Fig. 10 The critical lines $\overline{M R}, \overline{M Q}$, and $\overline{M P}$ are given by $a_{B}=0\left(a_{A} \leq 0\right)$, $a_{A}=0\left(a_{B} \leq 0\right)$, and $a_{A}=\widetilde{\mu} a_{B}\left(a_{B} \geq 0\right)$, respectively. The multicritical point $M$ locates at $\left(a_{A}, a_{B}\right)=(0,0)$.

Approaching the multicritical point along a straight line $\left(a_{A}, a_{B}\right)=(\varepsilon, r \varepsilon)$ with fixed $r$ inside the phase III, we find that the density vanishes as $\rho_{A, B} \sim \varepsilon^{\beta_{A, B}}$ with the order parameter exponents $\beta_{A}=2$ and $\beta_{B}=1$. Spatial fluctuations in the order parameter are easily incorporated into the mean field theory by including diffusion terms $\nabla^{2} \rho_{A, B}$ in the mean field equations. With those, one can show that the characteristic length scale diverges as $\xi_{A, B} \sim \varepsilon^{-v_{A, B}}$ with the correlation length exponent $v_{A}=v_{B}=1 / 2$. The characteristic time scale diverges with the length scale as $\tau_{A, B} \sim \xi_{A, B}^{z_{A}, B}$ with the dynamic exponent $z_{A}=z_{B}=2$.

We note that the critical exponents for the $B$ species are identical to the mean field DP exponents with $\beta=1, v=1 / 2$, and $z=2$ [1]. On the other hand, the $A$ species dynamics is dressed by the critical DP fluctuations and falls into the nonDP class. In the UCDP limit with $\widetilde{\mu}=0$, we have the opposite situation; $A$ species falls into the DP class with $\beta_{A}=1$ and $B$ species into the non-DP class with $\beta_{B}=1 / 2$ [7]. From the mean field analysis we conclude that the inhibitory coupling is relevant and that the ACDP forms an independent universality class distinct from the UCDP class.

More interesting is the critical density decay dynamics at the multicritical point. Putting $a_{A}=a_{B}=0$ in Eq. (1), after straightforward calculations, we obtain that the density decays algebraically in time as $\rho_{A} \sim t^{-\alpha_{A}}$ and $\rho_{B} \sim t^{-\alpha_{B}}$ with the exponent $\alpha_{B}=1$ and the variable exponent

$$
\alpha_{A}=\max \{2, \widetilde{\mu}\}
$$


At $\tilde{\mu}=2$, logarithmic corrections appear as $\rho_{A} \simeq$ $t^{-2}(2 \widetilde{\lambda} \ln t)^{-1}$ and $\rho_{B} \simeq t^{-1}(1+1 /(2 \ln t))$. In the normal scaling, we expect that the density decay exponent is given by $\alpha=\beta /(z v)$ [1], which does not hold in our model for $\tilde{\mu}>2$. This is another novel feature of the ACDP universality class.

The mean field characteristics may be realized on regular lattices in higher space dimensions than the upper critical dimension and also on various networks including complete graphs, random networks, and small-world networks [10]. Recently, the small-world network is found to be useful and efficient in studying mean field critical phenomena [11] and it reflects some of network aspects in real biological and social systems. In this Letter, we adopt the small-world networks to test our mean-field result and also to get the information on the upper critical dimension of our model.

The small-world network is characterized by the total number of sites $N$, the average coordination number $2 Z$, and the so-called rewiring probability $p_{r}$. It is constructed as follows [10]: First, $N$ sites are arranged along an onedimensional ring and each site is connected up to $Z$ neighboring sites. Second, each bond is rewired randomly with probability $p_{r}$. It interpolates the normal one-dimensional lattice at $p_{r}=0$ and the random network at $p_{r}=1$. In simulations, we choose $Z=10, p_{r}=0.5$, and $N \leq 2 \times 10^{6}$.

In networks, the concepts of dimensionality as well as length scale are meaningless. Botet et al. [12] suggested that a correlation volume, instead of length, $\xi_{V}$ be a proper scaling variable and the criticality be described by the correlation volume exponent $\bar{v}$ and the effective dynamic exponent $\bar{z}$. Moreover, they conjectured that the exponents are given by

$$
\overline{\mathrm{v}}=\mathrm{v}^{M F} d^{u} \quad \text { and } \quad \bar{z}=z^{M F} / d^{u}
$$

where $v^{M F}$ and $z^{M F}$ are the mean-field exponents and $d^{u}$ is the upper critical dimension. Although the conjecture has not been proved yet, it is shown to be valid for some equilibrium systems [11, 12]. We will use this relation to find the upper critical dimension of our system.

The phase boundary $\overline{M R}$ is given by a flat line of $p_{B}=p_{B}^{c}$, which is the critical probability of the uncoupled system. We perform Monte Carlo simulations starting with the fully occupied configuration of $B$ particles only. All numerical data shown below are obtained after average over, at least, several thousands of samples. As the particle density decays algebraically in time at criticality, we can easily locate $p_{B}^{c}=0.48505(5)$ where $\rho_{B} \sim t^{-\alpha_{B}}$ with $\alpha_{B}=0.98(5)$. This confirms that the critical line $\overline{M R}$ belongs to the mean-field DP class.

Once $p_{B}^{c}$ being found, the multicritical point $M$ can be located by examining the decay dynamics of $\rho_{A}$, starting from the fully occupied configuration of $A$ particles, as one varies $p_{A}$ with $p_{B}=p_{B}^{c}$ fixed. For the coupling constants, we fix $\lambda=0.5$ and vary $\mu$. We first study the UCDP limit at $\mu=0$. In Fig. 2 (a), the particle density decay is presented at the multicritical point at $p_{A}^{c}=0.3202(1)$. The density decay exponents are estimated as $\alpha_{A}=0.98(3)$ and $\alpha_{B}=0.49(2)$ as indicated
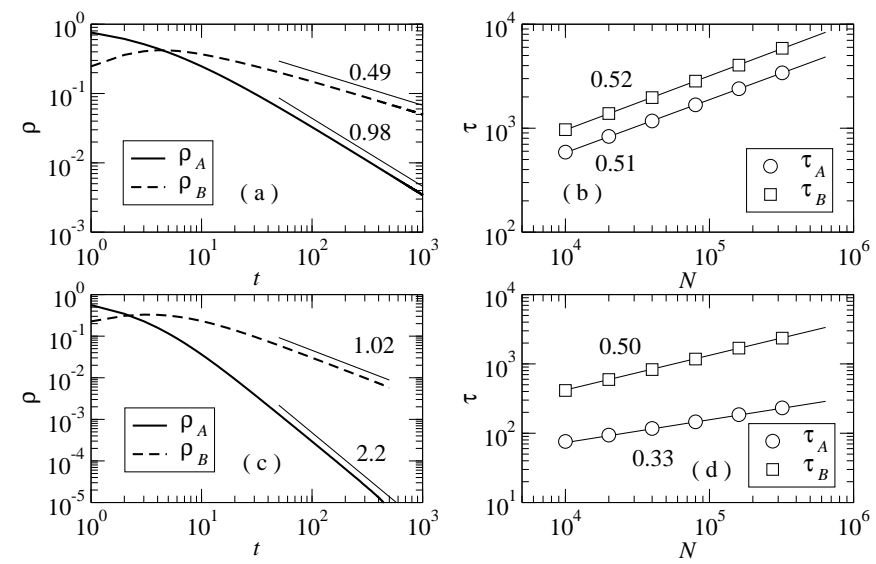

FIG. 2: (a) Density decay $\left(N=2 \times 10^{6}\right)$ and (b) characteristic time scale at $\mu=0$ and $p_{A}=0.3202$. (c) Density decay $\left(N=2 \times 10^{6}\right)$ and (d) characteristic time scale at $\mu=1.0$ and $p_{A}=0.2902$.

by straight lines in the plot. Note that the results are consistent with the mean field results of the UCDP [7]. In Fig. 2](b), we present the scaling of the characteristic time $\tau$ at which the survival probability becomes $1 / 2$. It grows algebraically with the total number of sites $N$ with the effective dynamic exponent $\bar{z}_{A}=0.51(2)$ and $\bar{z}_{B}=0.52(2)$ as indicated by straight lines. Using Eq. (3) and the mean field dynamic exponents $z_{A}=z_{B}=2$ [7], we obtain that the upper critical dimensionality is given by $d^{u}=4$ for both species, which is consistent with the prediction for the UCDP class [7].

Turning on the inhibitory coupling $(\mu \neq 0)$, we find different scaling exponents. In Fig. 2] (c), the density decay is shown at the multicritical point $p_{A}^{c}=0.2902(2)$ at $\mu=1$. As predicted in our mean field theory, the estimated decay exponent $\alpha_{B}=1.02(3)$ is consistent with the mean-field DP value and $\alpha_{A}=2.20(5)$ represents the non-DP class. We also estimate the effective dynamic exponents $\bar{z}_{A}=0.33(1) \simeq 1 / 3$ and $\bar{z}_{B}=0.50(1)$ in Fig. 22(d). Since $z^{M F}=2$ for both species, Eq. (3) suggests that the two species have different upper critical dimensions, i.e. $d_{A}^{u}=6$ and $d_{B}^{u}=4$. It is remarkable to see that the two interacting species begin to show the mean field critical behaviors at different dimensionality. A simple power counting for coupled Langevin equations obtained from Eq. (1) by adding diffusion terms and DP-type multiplicative noises reproduces the above results, but more efforts are needed to understand this peculiar property both analytically and numerically.

We performed the similar analysis for various values of $\mu$. The results for the decay exponents and the effective dynamic exponents are presented in Fig. 3 The numerical results are in good agreement with the mean field results. Near $\mu=0$, e.g., at $\mu=0.25$, the exponents seem to deviate from the mean field values. These are attributed to crossovers due to the nearby UCDP fixed point. The mean field theory and the numerical results suggest that the ACDP process with $\mu \neq 0$ form a distinct universality class different from the UCDP class.

The previous study poses a question on the relevance of the 

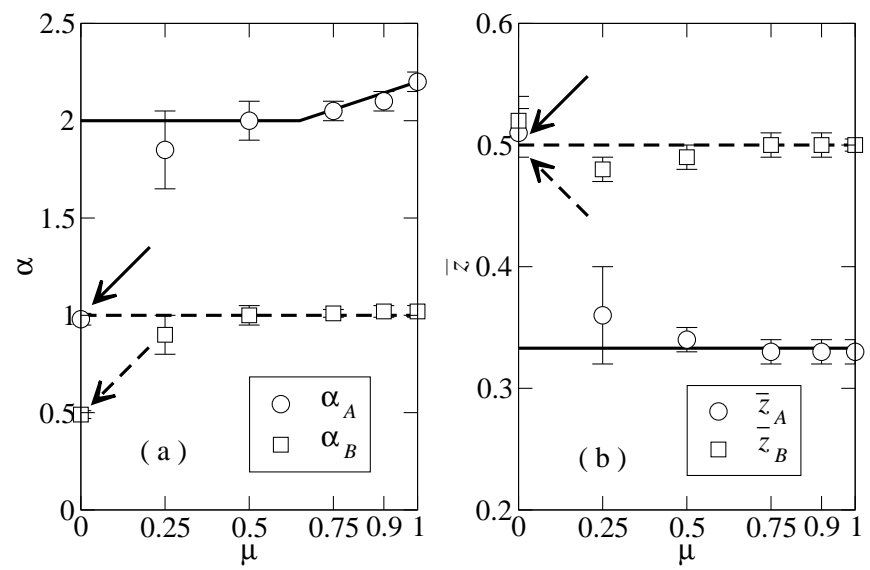

FIG. 3: (a) The density decay exponent and (b) the effective dynamic exponents at the multicritical points. The solid (dashed) lines are guides to eyes and indicate the mean field results for the $A(B)$ species of the ACDP process. The solid (dashed) arrows point toward the corresponding exponent values of the mean field UCDP class.
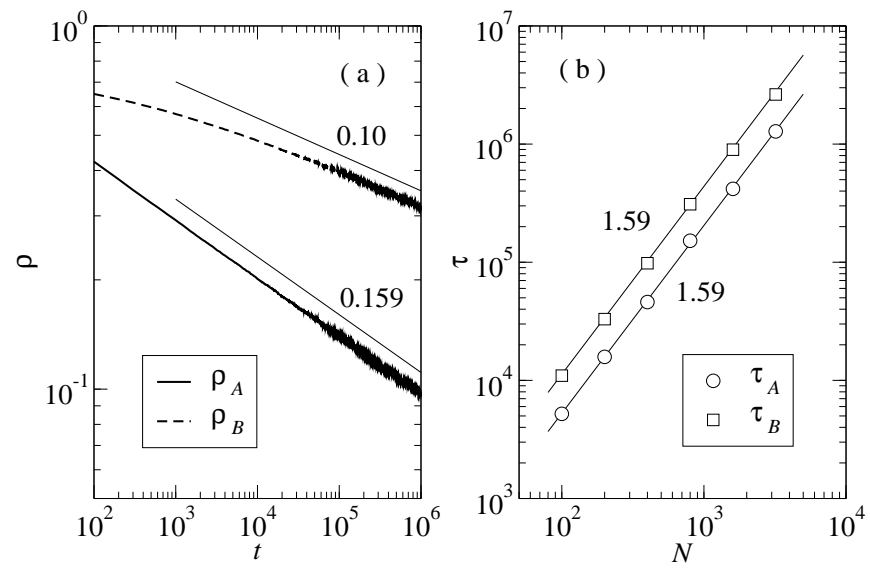

FIG. 4: (a) Density decay at the multicritical point of the ACDP model with $\lambda=0.2$ and $\mu=0.2$ on the one-dimensional lattice with $N=10^{5}$ sites. (b) Characteristic time scales at the multicritical point.

inhibitory coupling at lower dimensions [7]. We check the multicritical behavior of our model on the one-dimensional lattice. We fix the coupling constants $\lambda=0.2$ and $\mu=0.2$. Numerical simulations show that the multicritical point is located at $\left(p_{A}, p_{B}\right)=(0.14627(2), 0.23267)$. The density decay and the characteristic time scales are presented in Fig. 3 The decay exponents are estimated as $\alpha_{A}=0.159(5)$ and $\alpha_{B}=0.10(2)$, which are consistent with the results for the UCDP class in one dimension [7]. The dynamic exponents are esimated as $z_{A} \simeq z_{B} \simeq 1.59(2)$, which are consistent with the DP value. These results suggest that the ACDP process belongs to the UCDP class in one dimension. That is, the inhibitory coupling is irrelevant in one dimension in the contrary to the higher dimensional cases.
In summary, we have introduced the ACDP process of two particle species and investigated its multicritical behaviors. In the mean field theory, we show that the inhibitory coupling is relevant and the ACDP process forms the different universality class distinct from the UCDP process: The $B$ species displays the DP type critical behavior while the $A$ species the dressed non-DP type critical behavior. Furthermore, the critical dynamics of the $A$ species is anomalous with the variable critical exponent. The mean field results are confirmed by the numerical simulation studies of the ACDP on the small-world networks. Using the conjecture in Eq. (3), we also find that the upper critical dimensionality for each species is given by $d_{A}^{u}=6$ and $d_{B}^{u}=4$. On the other hand, the numerical simulation studies in one dimension show that the inhibitory coupling is irrelevant and the ACDP process belongs to the UCDP class: The $A$ species displays the DP type critical behavior while the $B$ species the dressed non-DP type critical behavior.

For future works, we suggest that it would be interesting to study the model in higher dimensional lattices to find the dimensionality at which the inhibitory coupling becomes relevant and to cofirm the upper critical dimensionalities. The multi-species generalization would be also interesting [13]. As an application, the phase transition of the ACDP process on general complex networks may yield richer critical dynamics.

This work was supported by Korea Research Foundation Grant (KRF-2004-041-C00139).

[1] H. Hinrichsen, Adv. Phys. 49, 815 (2000).

[2] H. K. Janssen, Z. Phys. B 42, 151 (1981).

[3] P. Grassberger, Z. Phys. B 47, 365 (1982).

[4] T. E. Harris, Ann. Prob. 2, 969 (1974).

[5] H. K. Janssen, Phys. Rev. Lett. 78, 2890 (1997); J. Stat. Phys. 103, 801 (2001).

[6] S. Kwon and H. Park, J. Korean Phys. Soc. 38, 490 (2001); Phys. Rev. E 69, 066125 (2004).

[7] U. C. Täuber, M. J. Howard, and H. Hinrichsen, Phys. Rev. Lett. 80, 2165, (1998); Y. Y. Goldschmidt, ibid. 81, 2178 (1998).

[8] Y. Y. Goldschmidt, M. J. Howard, H. Hinrichsen, and U. C. Täuber, Phys. Rev. E 59, 6381 (1999).

[9] U. Alon, M. R. Evans, H. Hinrichsen, and D. Mukamel, Phys. Rev. Lett. 76, 2746 (1996); Phys. Rev. E 57, 4997 (1998).

[10] D. J. Watts and S. H. Strogatz, Nature (London) 393, 440 (1998).

[11] B. J. Kim, H. Hong, P. Holme, G. S. Jeon, P. Minnhagen, and M. Y. Choi, Phys. Rev. E 64, 056135 (2001); H. Hong, B. J. Kim, and M. Y. Choi, Phys. Rev. E 66, 018101 (2002).

[12] R. Botet, R. Jullien, and P. Pfeuty, Phys. Rev. Lett. 49, 478 (1982).

[13] The mean field exponents for the multi-species ACDP display an interesting Fibonacci sequence. 